Canad. Math. Bull. Vol. 56 (3), 2013 pp. 640-646

http://dx.doi.org/10.4153/CMB-2012-017-x

(c) Canadian Mathematical Society 2012

\title{
Regulator Indecomposable Cycles on a Product of Elliptic Curves
}

\author{
İnan Utku Türkmen
}

Abstract. We provide a novel proof of the existence of regulator indecomposables in the cycle group $C H^{2}(X, 1)$, where $X$ is a sufficiently general product of two elliptic curves. In particular, the nature of our proof provides an illustration of Beilinson rigidity.

\section{Introduction}

Let $X$ be a smooth projective algebraic manifold of dimension $n$ and let $C H^{k}(X, m)$ be the higher Chow group of cycles, introduced in [1]. Our interest is the case $m=1$, where an abridged definition of $C H^{k}(X, 1)$ goes as follows. A class $\gamma \in C H^{k}(X, 1)$ is represented as a formal sum $\gamma=\sum\left(g_{j}, Z_{j}\right)$ of non-zero rational functions $g_{j}$ on irreducible subvarieties $Z_{j}$ of codimension $k-1$ in $X$ such that $\sum \operatorname{div} g_{j}=0$. One then quotients out by the image of Tame symbols to arrive at the group $C H^{k}(X, 1)$. The group of decomposable cycles, denoted by $C H_{\mathrm{dec}}^{k}(X, 1)$, is defined to be the image of the intersection product $C H^{1}(X, 1) \otimes C H^{k-1}(X) \rightarrow C H^{k}(X, 1)$, where in this situation $C H^{1}(X, 1)=\mathbb{C}^{\times}([1])$.

With this definition, decomposable cycles are represented by those with (nonzero) constant rational functions $g_{j}$. The corresponding group of indecomposables is the quotient $C H_{\text {ind }}^{k}(X, 1):=C H^{k}(X, 1) / C H_{\text {dec }}^{k}(X, 1)$. There are a number of results centered around constructing indecomposable higher Chow cycles $[3,5-8]$, and in some cases countably infinite generation results for group of indecomposables are obtained $[3,7]$. One of the methods to detect indecomposable cycles is regulator indecomposability, introduced in [4]. A higher Chow cycle $\zeta=\sum\left(g_{j}, Z_{j}\right)$ is called regulator indecomposable if the current defined by its real regulator

$$
r(\zeta)(\omega)=\frac{1}{(2 \pi \sqrt{-1})^{d-k+1}} \sum\left(\int_{Z_{j}-Z_{j}^{\text {sing }}} \omega \log |f|\right)
$$

is nonzero for some real $d$-closed test form $\omega$ of Hodge type $(1,1)$, with class in $H^{1,1}\left(E_{1} \times E_{2}, \mathbb{R}\right)$ orthogonal to $\mathrm{Hg}^{1}\left(E_{1} \times E_{2}\right) \otimes \mathbb{R}$. A regulator indecomposable cycle is clearly indecomposable. The proof of [4, Theorem 1] (pertaining to the existence

Received by the editors July 7, 2011; revised March 28, 2012.

Published electronically July 16, 2012.

The author was partially supported by the Scientific and Technological Research Council of Turkey through the International Research Fellowship Programme and the National Scholarship Programme for Ph.D. Students.

AMS subject classification: 14C25.

Keywords: real regulator, regulator indecomposable, higher Chow group, indecomposable cycle. 
of regulator indecomposables in the higher cycle group $C H^{2}(X, 1)$, where $X$ is a sufficiently general product of two elliptic curves) contains an error that was subsequently fixed in [2] using an entirely different set of techniques. The purpose of this paper is to prove this theorem in the spirit of the original techniques in [4].

\section{Notation}

Throughout this paper, $X$ is assumed to be a projective algebraic manifold. For a subring $\mathbb{A} \subset \mathbb{R}$, put $\mathbb{A}(k)=\mathbb{A}(2 \pi \sqrt{-1})^{k}$. Our notation is compatible with [4].

\section{Constructing a Higher Chow Cycle}

For $j=1,2$ let $E_{j} \subset \mathbb{P}^{2}$ be elliptic curves defined by the Weierstrass equations

$$
F_{j}=y_{j}^{2}-x_{j}^{3}+b_{j} x_{j}+c_{j} \quad \text { and } \quad X=V\left(\bar{F}_{1}, \bar{F}_{2}\right) \simeq E_{1} \times E_{2} .
$$

Clearly $X$ varies with $t=\left(b_{1}, c_{1}, b_{2}, c_{2}\right)$. We consider the family $X:=V\left(\bar{F}_{1}, \bar{F}_{2}\right) \subset$ $\left(C^{4} \times \mathbb{P}^{2} \times \mathbb{P}^{2}\right.$. Sufficiently general $X$ means, $X=X_{t}$ in a transcendental sense, with $t$ outside a suitable countable union of proper Zariski closed subsets.

Let $D$ be the curve of intersection of $X$ with the hypersurface given by $V\left(s_{1} t_{1}+s_{2} t_{2}\right)$, where $\left[s_{0}, s_{1}, s_{2}\right]$ and $\left[t_{0}, t_{1}, t_{2}\right]$ are homogeneous coordinates of $\mathrm{P}^{2} \supset E_{1}$ and $\mathrm{P}^{2} \supset E_{2}$ respectively, as in [4], with $x_{1}=\frac{s_{1}}{s_{0}}, x_{2}=\frac{t_{1}}{t_{0}}, y_{1}=\frac{s_{2}}{s_{0}}, y_{2}=\frac{t_{2}}{t_{0}}$. Under the Segre embedding $s: \mathbb{P}^{2} \times \mathbb{P}^{2} \hookrightarrow \mathbb{P}^{8}$, given by

$$
s:\left[s_{0}, s_{1}, s_{2} ; t_{0}, t_{1}, t_{2}\right] \mapsto\left[s_{0} t_{0}, s_{1} t_{0}, s_{2} t_{0}, s_{0} t_{1}, s_{1} t_{1}, s_{2} t_{1}, s_{0} t_{2}, s_{1} t_{2}, s_{2} t_{2}\right],
$$

$D$ corresponds to a $\mathbb{P}^{7} \subset \mathbb{P}^{8}$ intersecting with $X$. By [4, Lemma 2.2], D is smooth and irreducible for general $t$.

In [4] the function $f=x_{1}-\sqrt{-1}$ and the form $\omega:=\left(\frac{d x_{1}}{y_{1}} \wedge \frac{d \bar{x}_{2}}{\bar{y}_{2}}+\frac{d \bar{x}_{1}}{\bar{y}_{1}} \wedge \frac{d x_{2}}{y_{2}}\right)$ in affine coordinates are considered, and it is claimed that

$$
\int_{D} \omega \log |f| \neq 0
$$

For general $X, w \in\left(H g^{1}(X) \oplus \mathbb{R}\right)^{\perp}\left(\right.$ see [4, Lemma 2.5]), where $H^{1}(X)$ denotes the group of Hodge cycles of codimension 1 on $X$. This claim is proved by means of two deformation arguments; first, deforming $D_{t}$ from generic point $t=\left(b_{1}, c_{1}, b_{2}, c_{2}\right)$ to $t=\left(b_{1}, 0, b_{2}, 0\right)$ and then considering the limit case as $\left(b_{1}, b_{2}\right) \mapsto(0,0)$. However, there is an error in the second deformation argument. We discuss this error briefly below.

When $t=\left(b_{1}, 0, b_{2}, 0\right)$, we have $X=E_{1} \times E_{2}$ where $E_{j}$ is given by the equation $y_{j}^{2}=x_{j}^{3}+b_{j} x_{j}$ and $D_{t}=X \cap V\left(x_{1} x_{2}+y_{1} y_{2}=0\right)$. Notice that on $D_{t}$ we have

$$
x_{1}^{2} x_{2}^{2}=y_{1}^{2} y_{2}^{2}=x_{1} x_{2}\left(x_{1}^{2}+b_{1}\right)\left(x_{2}^{2}+b_{2}\right)
$$

and we can decompose

$$
D_{t}=\left(E_{1} \times[1,0,0]\right)+\left([1,0,0] \times E_{2}\right)+\grave{D_{t}},
$$


where $x_{1} x_{2}=\left(x_{1}^{2}+b_{1}\right)\left(x_{2}^{2}+b_{2}\right)$ on $\grave{D_{t}}$. We can cancel a factor of $x_{1} x_{2}$, corresponding to the curve $\left(E_{1} \times[1,0,0]\right)+\left([1,0,0] \times E_{2}\right)$, since the pull back of the real 2-form $\omega$ to this component is zero. Hence we have

$$
\int_{D_{t}} \omega \log |f|=\int_{\dot{D}_{t}} \omega \log |f|
$$

and we are left with the family $\sum:=\bigcup_{t \in U} \grave{D_{t}}$ for some neighbourhood $U$ of $t$.

In the second degeneration argument, $\left(b_{1}, b_{2}\right) \mapsto(0,0)$, we have $X=E_{1} \times E_{2}$, where the elliptic curves $E_{j}$ themselves degenerate to $y_{j}^{2}=x_{j}^{3}$, and we can decompose $\grave{D}_{t}$ into three pieces $D$, $\left(E_{1} \times[1,0,0]\right)$ and $\left([1,0,0] \times E_{2}\right)$ where $D=D \cap V\left(x_{1} x_{2}-1\right)$. Moreover, we have $x_{1} x_{2}=x_{1}^{2} x_{2}^{2}$ on $D$, but this time we cannot cancel the factor $x_{1} x_{2}$, since the real 2-form $\omega$ acquires singularities and contributions to the real regulator from different parts cancel each other.

We will keep track of this deformation and show that the contributions to real regulator from the parts $\overparen{D}$ and $\left(E_{1} \times[1,0,0]\right)$ cancel each other by direct calculation of integrands in the limit case. To see this, and for notational simplicity, let us take $b_{1}=b_{2}=\epsilon$. On $\grave{D}$, we have $x_{1} x_{2}=\left(x_{1}^{2}+\epsilon\right)\left(x_{2}^{2}+\epsilon\right)$ and $x_{1}$ is a local coordinate on a Zariski open subset of each irreducible component of $\grave{D}$ (provided we discard the component $[1,0,0] \times E_{2}$ when $b_{1}=b_{2}=0$, which we can do, as this amounts to the observation that $\log |f|=\log \left|x_{1}-\sqrt{-1}\right|=0$ there). We now apply some first order approximations. For small values of $|\epsilon|$, we have $x_{1} x_{2} \approx x_{1}^{2} x_{2}^{2}$, and if $x_{1} x_{2} \neq 0$, then $x_{1} x_{2}=1$, and $x_{2} \approx x_{1}^{-1}$ is a solution. On the other hand, regarding $E_{1} \times[1,0,0]$, we look at small values of $\left|x_{2}\right|$ and we get $x_{1} x_{2} \approx \epsilon\left(x_{1}^{2}+\epsilon\right) \approx \epsilon x_{1}^{2}$, and $x_{2} \approx \epsilon x_{1}$ is a solution. Clearly, the former one limits to $D$ and the latter to $E_{1} \times[1,0,0]$. To reiterate, we can discard the other component $[1,0,0] \times E_{2}$. So we will compute the limiting integral of $\log \left|x_{1}-\sqrt{-1}\right| \omega$ for these two approximate solutions.

Consider

$$
\omega=\left(\frac{d x_{1}}{\sqrt{x_{1}^{3}+\epsilon x_{1}}}\right) \wedge \overline{\left(\frac{d x_{2}}{\sqrt{x_{2}^{3}+\epsilon x_{2}}}\right)}+\overline{\left(\frac{d x_{1}}{\sqrt{x_{1}^{3}+\epsilon x_{1}}}\right)} \wedge\left(\frac{d x_{2}}{\sqrt{x_{2}^{3}+\epsilon x_{2}}}\right)
$$

For $x_{2}=x_{1}^{-1}, d x_{2}=-x_{1}^{-2} d x_{1}$. Plugging this in above equation,

$\omega=\left(\frac{d x_{1}}{\left(x_{1}^{3}+\epsilon x_{1}\right)^{\frac{1}{2}}}\right) \wedge \overline{\left(\frac{-x_{1}^{-2} d x_{1}}{\left(x_{1}^{-3}+\epsilon x_{1}^{-1}\right)^{\frac{1}{2}}}\right)}+\overline{\left(\frac{d x_{1}}{\left(x_{1}^{3}+\epsilon x_{1}\right)^{\frac{1}{2}}}\right)} \wedge\left(\frac{-x_{1}^{-2} d x_{1}}{\left(x_{1}^{-3}+\epsilon x_{1}^{-1}\right)^{\frac{1}{2}}}\right)$.

Arranging the terms, we get

$$
\begin{aligned}
\omega & =-\frac{d x_{1}}{x_{1}^{\frac{1}{2}}\left(x_{1}^{2}+\epsilon\right)^{\frac{1}{2}}} \wedge \frac{d \overline{x_{1}}}{\bar{x}_{1}^{\frac{1}{2}} \overline{\left(1+\epsilon x_{1}^{2}\right)^{\frac{1}{2}}}}-\frac{d \overline{x_{1}}}{\bar{x}_{1}^{\frac{1}{2}} \overline{\left(x_{1}^{2}+\epsilon\right)^{\frac{1}{2}}}} \wedge \frac{d x_{1}}{x_{1}^{\frac{1}{2}}\left(1+\epsilon x_{1}^{2}\right)^{\frac{1}{2}}} \\
& =\left(\frac{-1}{x_{1}^{\frac{1}{2}}\left(x_{1}^{2}+\epsilon\right)^{\frac{1}{2}} \bar{x}_{1}^{\frac{1}{2}} \overline{\left(1+\epsilon x_{1}^{2}\right)^{\frac{1}{2}}}}+\frac{1}{\bar{x}_{1}^{\frac{1}{2}} \overline{\left(x_{1}^{2}+\epsilon\right)^{\frac{1}{2}} x_{1}^{\frac{1}{2}}\left(1+\epsilon x_{1}^{2}\right)^{\frac{1}{2}}}}\right) d x_{1} \wedge d \overline{x_{1}} \\
& =\left(\frac{x_{1}^{\frac{1}{2}}\left(x_{1}^{2}+\epsilon\right)^{\frac{1}{2}} \bar{x}_{1}^{\frac{1}{2}} \overline{\left(1+\epsilon x_{1}^{2}\right)^{\frac{1}{2}}}-\overline{x_{1}} \frac{1}{\left(1+\epsilon x_{1}^{2}\right)^{\frac{1}{2}}} x_{1}^{\frac{1}{2}}\left(1+\epsilon x_{1}^{2}\right)^{\frac{1}{2}}}{\left|x_{1}\right|\left|1+\epsilon x_{1}^{2}\right|\left|x_{1}^{2}+\epsilon \| x_{1}\right|}\right) d x_{1} \wedge d \overline{x_{1}} .
\end{aligned}
$$


Taking the limit as $\epsilon \rightarrow 0$, we have

$$
\omega=\left(\frac{x_{1}^{\frac{3}{2}} \bar{x}_{1}^{\frac{1}{2}}-x_{1}^{\frac{1}{2}} \bar{x}_{1}^{\frac{3}{2}}}{\left|x_{1}\right|^{4}}\right) d x_{1} \wedge d \overline{x_{1}}=\left(\frac{x_{1}-\overline{x_{1}}}{\left|x_{1}\right|^{3}}\right) d x_{1} \wedge d \overline{x_{1}} \text { on } D^{\prime \prime}
$$

As $\epsilon \rightarrow 0, x_{2}=x_{1}^{-1}$ has limit $D$ and

$$
\log |f| \omega \rightarrow \log \left|x_{1}-\sqrt{-1}\right|\left(\frac{x_{1}-\overline{x_{1}}}{\left|x_{1}\right|^{3}}\right) d x_{1} \wedge d \overline{x_{1}}
$$

Let us consider the latter approximation $x_{2}=\epsilon x_{1}$. When $x_{2}=\epsilon x_{1}$, we have $d x_{2}=\epsilon d x_{1}$. Plugging these relations into equation (3.1), we get;

$$
\begin{aligned}
\omega & =\left(\frac{d x_{1}}{\left(x_{1}^{3}+\epsilon x_{1}\right)^{\frac{1}{2}}}\right) \wedge \overline{\left(\frac{\epsilon d x_{1}}{\left(\epsilon^{3} x_{1}^{3}+\epsilon^{2} x_{1}\right)^{\frac{1}{2}}}\right)}+\overline{\left(\frac{d x_{1}}{\left(x_{1}^{3}+\epsilon x_{1}\right)^{\frac{1}{2}}}\right)} \wedge\left(\frac{\epsilon d x_{1}}{\left(\epsilon^{3} x_{1}^{3}+\epsilon^{2} x_{1}\right)^{\frac{1}{2}}}\right) \\
& =\left(\frac{d x_{1}}{\left(x_{1}^{3}+\epsilon x_{1}\right)^{\frac{1}{2}}}\right) \wedge \frac{\bar{\epsilon} d \overline{x_{1}}}{\overline{\left(\epsilon^{3} x_{1}^{3}+\epsilon^{2} x_{1}\right)^{\frac{1}{2}}}}+\frac{d \overline{x_{1}}}{\overline{\left(x_{1}^{3}+\epsilon x_{1}\right)^{\frac{1}{2}}}} \wedge\left(\frac{\epsilon d x_{1}}{\left(\epsilon^{3} x_{1}^{3}+\epsilon^{2} x_{1}\right)^{\frac{1}{2}}}\right) \\
& =\left(\frac{\bar{\epsilon}}{\left(x_{1}^{3}+\epsilon x_{1}\right)^{\frac{1}{2}} \overline{\left(\epsilon^{3} x_{1}^{3}+\epsilon^{2} x_{1}\right)^{\frac{1}{2}}}}-\frac{\epsilon}{\overline{\left(x_{1}^{3}+\epsilon x_{1}\right)^{\frac{1}{2}}}\left(\epsilon^{3} x_{1}^{3}+\epsilon^{2} x_{1}\right)^{\frac{1}{2}}}\right) d x_{1} \wedge d \overline{x_{1}} .
\end{aligned}
$$

Taking the limit as $\epsilon \rightarrow 0$, we get

$$
\omega=\left(\frac{1}{x_{1}^{\frac{3}{2}} \overline{x_{1}} \frac{1}{2}}-\frac{1}{\bar{x}_{1}^{\frac{3}{2}} x_{1}^{\frac{1}{2}}}\right) d x_{1} \wedge d \overline{x_{1}}=\left(\frac{\overline{x_{1}}-x_{1}}{\left|x_{1}\right|^{3}}\right) d x_{1} \wedge d \overline{x_{1}} \text { on } E_{1} \times[1,0,0] .
$$

In the limit as $\epsilon \rightarrow 0, x_{2}=\epsilon x_{1}$ has limit $E_{1} \times[1,0,0]$ and

$$
\log |f| \omega \rightarrow \log \left|x_{1}-\sqrt{-1}\right|\left(\frac{\overline{x_{1}}-x_{1}}{\left|x_{1}\right|^{3}}\right) d x_{1} \wedge d \overline{x_{1}}
$$

(As a reminder, when $b_{1}=b_{2}=0, E_{1}=E_{2}$ are (singular) rational curves.) In the limit, the contributions of these parts to the real regulator cancel one another.

In order to solve this problem, we consider the function $f=x_{1}^{2} x_{2}-\sqrt{-1}$ and the same form $\omega$. Note that for the solution $x_{2}=\epsilon x_{1}$, which limits to the component $E_{1} \times[1,0,0], \log \left|x_{1}^{2} x_{2}-\sqrt{-1}\right|=\log \left|\epsilon x_{1}^{3}-\sqrt{-1}\right|$, goes to zero as $\epsilon \rightarrow 0$, so in the limit, $\log |f| \omega$ vanishes. However for the second solution $x_{2}=x_{1}^{-1}$, we have $\log \left|x_{1}^{2} x_{2}-\sqrt{-1}\right|=\log \left|x_{1}-\sqrt{-1}\right|$. In the limit we get the component $D$ and recover the function $\log \left|x_{1}-\sqrt{-1}\right|$ introduced in [4], which contributes to the real regulator nontrivially.

Since the function $f=x_{1}^{2} x_{2}-\sqrt{-1}$ is not linear as in [4], it requires a more complicated and different argument to complete the tuple $(f, D)$ to a higher Chow cycle.

Let $E_{j \text {,tor }}$ denote the set of torsion points on $E_{j}$. We define $D_{\text {tor }}:=\left\{E_{1, \text { tor }} \times E_{2}\right\} \cap D$. For sufficiently general $X, D$ is a smooth irreducible curve. Moreover, $E_{1, \text { tor }}$ is dense 
in $E_{1}$ and $D \subset X=E_{1} \times E_{2}$ projects onto first factor, so $D_{\text {tor }}$ is dense in $D$. In projective coordinates the function $f$ is given by

$$
f=x_{1}^{2} x_{2}+\sqrt{-1}=\frac{s_{1}^{2} t_{1} t_{0}+s_{0}^{2} t_{0}^{2} \sqrt{-1}}{s_{0}^{2} t_{0}^{2}} .
$$

Under the Segre embedding $f$ is a quotient of two quadrics

$$
Q_{1,0}=s_{1}^{2} t_{1} t_{0}+s_{0}^{2} t_{0}^{2} \sqrt{-1}=\left(s_{1} t_{1}\right)\left(s_{1} t_{0}\right)+\left(s_{0} t_{0}\right)^{2} \sqrt{-1}
$$

and

$$
Q_{2,0}=s_{0}^{2} t_{0}^{2}=\left(s_{0} t_{0}\right)^{2} .
$$

Counted with multiplicities, the divisor of $f$ along $D$ is given by

$$
\operatorname{div}(f)_{D}=V\left(Q_{1,0}\right) \cap D-V\left(Q_{2,0}\right) \cap D .
$$

Note that for a quadric $Q \in \mathbb{P}^{8}$, $\operatorname{deg}(Q \cap D)=36$. Consider the family of quadrics lying in a $\mathbb{P}^{7} \subset \mathbb{P}^{8}$ cutting out $D \subset E_{1} \times E_{2}$ under the Segre embedding. This family is a projective space of dimension 35 . Hence the family of quadrics passing through 35 general points of $D$ is zero dimensional. If we set $Q \cap D=\left\{p_{1}+\cdots+p_{36}\right\}$, and assume that $\left\{p_{1} \cdots p_{35}\right\} \in D_{\text {tor }}$, then $p_{36} \in D_{\text {tor }}$.

Let $q_{1}^{i} \cdots q_{36}^{i} \in \operatorname{div}_{D}\left(Q_{i, 0}\right)$. Since $D_{\text {tor }}$ is dense in $D$, for any given collection of analytic neighborhoods $\left\{U_{i}\right\}$ around $q_{i}$ for $i=1 \cdots 36$, we can find 36 points $p_{1}^{i}, \ldots, p_{36}^{i} \in D_{\text {tor }}$, lying in a quadric intersected with $D$, such that $p_{j}^{i} \in U_{i}$. By the above argument these points define quadratic functions $Q_{i, n}$ for $i=1,2$ and $\widetilde{f}_{n}=Q_{1, n} / Q_{2, n}$ such that $p_{1}^{i}, \ldots, p_{36}^{i} \in \operatorname{div}_{D}\left(\widetilde{f}_{n}\right) \subset D_{\text {tor }}$; moreover, using the fact that if $h_{1}, h_{2} \in \mathbb{C}^{\times}$with $\operatorname{div}\left(h_{1}\right)=\operatorname{div}\left(h_{2}\right)$ then $h_{1}=c \cdot h_{2}$ for some $c \in \mathbb{C}^{\times}$, we can arrange for $\lim _{n \rightarrow \infty} \widetilde{f}_{n}=f$.

Let $\Delta_{j}$ be a small open polydisk in the space of quadratic polynomials in $\mathbb{C}\left[z_{0}, \ldots, z_{7}\right]$ centered at 0 for $j=1,2$. Then for $t \in \Delta:=\Delta_{1} \times \Delta_{2}$, one has a corresponding function $f_{t}=Q_{1, t} / Q_{2, t}$ with $f_{0}=Q_{1,0} / Q_{2,0}=f$.

Note that the set

$$
\bigcup_{t \in \Delta}\left|\operatorname{div}\left(f_{t}\right)\right|
$$

has real codimension $\geq 2$ in $\Delta \times D$. Considering $\epsilon$ tubular neighborhoods in $\Delta \times$ $D$ about this set and applying standard estimates as $\epsilon \mapsto 0^{+}$, we conclude that the integral $\int_{D} \log \left|f_{t}\right| \omega$ varies continuously with $t \in \Delta$.

We may assume that

$$
\int_{D} \log \left|f_{t}\right| \omega \neq 0, \quad \forall t \in \Delta .
$$

Since $\Delta$ parameterizes all quadratic quotients in a neighborhood of $(0,0) \in \Delta$, then for large enough $n$ we will have $\widetilde{f}_{n}=f_{t}$ for some $t \in \Delta$. Therefore

$$
\left|\int_{D} \log \right| f\left|\omega-\int_{D} \log \right| \widetilde{f}_{n}|\omega|<\epsilon
$$


for any small $\epsilon>0$ and large enough $n$ dependent on $\epsilon$.

The divisor of $\tilde{f}$ along $D$ can be written as

$$
\operatorname{div}_{D}(\tilde{f})=\sum_{j} n_{j}\left(p_{j} \times q_{j}\right) \in D_{\text {tor }}, \quad \text { where } n_{j} \in \mathbb{Z} \text { and } \sum_{j} n_{j}=0
$$

Let $e_{1}$ denote the identity element on $E_{1}$. By our construction, the $p_{j}$ 's are torsion points, so $m_{j} p_{j} \sim_{\text {rat }} m_{j} e_{1}$ for some $m_{j}$ (i.e., there exist rational functions $h_{j} \in$ $\mathbb{C}\left(E_{1}\right)^{\times}$such that $\left.\operatorname{div}_{E_{1}}\left(h_{j}\right)=m_{j} e_{1}-m_{j} p_{j}\right)$. Then for $m=\prod_{j} m_{j}$, we have $m p_{j} \sim_{\text {rat }}$ $m e_{1}$ for all $j$. So we likewise have rational functions $h_{j} \in \mathbb{C}\left(E_{1} \times q_{j}\right)^{\times}$such that $\operatorname{div}_{E_{1} \times q_{j}}\left(h_{j}\right)=m\left(e_{1} \times q_{j}\right)-m\left(p_{j} \times q_{j}\right)$. Consider the precycle $\left(\widetilde{f}^{m}, D\right)+\left\{h_{j}^{n_{j}}, E_{1} \times q_{j}\right\}_{j}$ :

$$
\begin{aligned}
& \operatorname{div}_{D}\left(\tilde{f}^{m}\right)+\sum_{j} \operatorname{div}_{E_{1} \times q_{j}}\left(h_{j}^{n_{j}}\right) \\
& =\sum_{j} m n_{j}\left(p_{j} \times q_{j}\right)+\sum_{j}\left(m n_{j}\left(e_{1} \times q_{j}\right)-m n_{j}\left(p_{j} \times q_{j}\right)\right) \\
& =\sum_{j} m n_{j}\left(e_{1} \times q_{j}\right):=\xi .
\end{aligned}
$$

The remaining term $\xi$ is the divisors of the functions $\widetilde{f}$ and $\left\{\left(h_{j}\right)\right\}_{j}$, hence it is rationally equivalent to zero on $E_{1} \times E_{2}$. The projection of $\xi$ to the second factor, $\operatorname{Pr}_{2, *}(\xi)$, is rationally equivalent to zero on $E_{2}$. So there exists a rational function $g$ defined on $e_{1} \times E_{2}$ such that $\operatorname{div}_{e_{1} \times E_{2}}(g)=-\sum_{j} m n_{j}\left(e_{1} \times q_{j}\right)$. Let

$$
\gamma=\left(\widetilde{f}^{m}, D\right)+\left\{\left(h_{j}^{n_{j}}, E_{1} \times q_{j}\right)\right\}_{j}+\left(g, e_{1} \times E_{2}\right)
$$

Then

$$
\operatorname{div}_{D}\left(\widetilde{f}^{m}\right)+\sum_{j} \operatorname{div}_{E_{1} \times q_{j}}\left(h_{j}^{n_{j}}\right)+\operatorname{div}_{e_{1} \times E_{2}}(g)=0 .
$$

Hence $\gamma \in C H^{2}(X, 1 ;(0))$ is a higher Chow cycle.

Note that the curves $E_{1} \times q_{j}$ and $p_{j} \times E_{2}$ cannot support the real 2-form $\omega$. Therefore the contributions of the terms $\left\{\left(h_{j}^{n_{j}}, E_{1} \times q_{j}\right)\right\}_{j}+\left(g, e_{1} \times E_{2}\right)$ to the real regulator are zero $\left(\int_{E_{1} \times q_{j}} \log \left|h_{j}\right| \omega=0=\int_{e_{1} \times E_{2}} \log |g| \omega\right)$, so

$$
r(\gamma)(\omega)=\int_{D} \omega \log \left|\widetilde{f}^{m}\right| \neq 0
$$

That is, $\gamma \in C H^{2}(X, 1 ;(0))$ is regulator indecomposable, so it is indecomposable, and hence we have the following theorem.

Theorem 3.1 $C H_{\text {ind }}^{2}\left(E_{1} \times E_{2}, 1 ;(0)\right)$ is nontrivial for sufficiently general product $E_{1} \times E_{2}$ of elliptic curves $E_{1}$ and $E_{2}$. 
Acknowledgment This paper is part of the author's $\mathrm{PhD}$ thesis under the direction of Professor James D. Lewis from the University of Alberta, Department of Mathematical and Statistical Sciences. The author would like to express his gratitude to Professor Lewis for his supervision, encouragement, and support. The author would also like to thank the referee for his/her valuable comments which strengthened this paper substantially.

\section{References}

[1] S. Bloch, Algebraic cycles and higher K-theory. Adv. in Math. 61(1986), no. 3, 267-304. http://dx.doi.org/10.1016/0001-8708(86)90081-2 http://dx.doi.org/10.1016/0001-8708(86)90081-2

[2] X. Chen and J. D. Lewis, The Hodge-D-conjecture for K3 and abelian surfaces. J. Algebraic Geom. 14(2005), no. 2, 213-240. http://dx.doi.org/10.1090/S1056-3911-04-00390-X http://dx.doi.org/10.1090/S1056-3911-04-00390-X

[3] A. Collino, Griffith' infinitesimal invariant and higher K-theory on hyperelliptic Jacobians. J. Algebraic Geom. 6(1997), no. 3, 393-415.

[4] B. B. Gordon and J. D. Lewis, Indecomposable higher Chow cycles on products of elliptic curves. J. Algebraic Geom. 8(1999), no. 3, 543-567.

[5] J. D. Lewis, A note on indecomposable motivic cohomology classes. J. Reine Angew. Math. 485(1997), 161-172. http://dx.doi.org/10.1515/crll.1997.485.161

[6] S. J. M. Mildenhall, Cycles in a product of elliptic curves, and a group analogous to the class group. Duke Math. J. 67(1992), no. 2, 387-406. http://dx.doi.org/10.1215/S0012-7094-92-06715-9 http://dx.doi.org/10.1215/S0012-7094-92-06715-9

[7] S. J. Müller-Stach, Constructing indecomposable motivic cohomology classes on algebraic surfaces. J. Algebraic Geom. 6(1997), no. 3, 513-543.

[8] M. Spiess, On indecomposable elements of $K_{1}$ of a product of elliptic curves. $K$-Theory 17(1999), no. 4, 363-383. http://dx.doi.org/10.1023/A:1007739216643 http://dx.doi.org/10.1023/A:1007739216643

Department of Mathematics, Bilkent University, Ankara, Turkey, 06800

e-mail: turkmen@fen.bilkent.edu.tr 